Editora Omnis Scientia

ANAIS DA I JORNADA NORTE-NORDESTE DE ENGENHARIAS (ONLINE)

Volume 1

$1^{\text {a }}$ Edição

TRIUNFO - PE 


\section{Coordenador Científico}

Alex Aguiar da Silva

\section{Coordenador de Publicação}

Daniel Luís Viana Cruz

\section{Coordenadora do Evento}

Andréa Telino Gomes

\section{Organizadores}

Academics - Eventos acadêmicos online

Editora Omnis Scientia

Andréa Telino Gomes

Daniel Luís Viana Cruz

Alex Aguiar da Silva

\section{Palestrantes}

Alex Aguiar da Silva

André Búrigo Leite

Cecília Aguiar da Silva

Eugênio Bastos da Costa

Jorge Recarte Henriquez Guerrero

Renan Ferreira da Rocha

\section{Avaliadores}

Alex Aguiar da Silva

Helio Cardoso Martim

Péricles Felipe Bastos Gomes

\section{Imagem de Capa}

Freepik

\section{Revisão}

Os autores

\section{(9) $\mathbb{\oplus \Theta \Theta}$}

Este trabalho está licenciado com uma Licença Creative Commons - Atribuição-NãoComercialSemDerivações 4.0 Internacional.

O conteúdo abordado nos artigos, seus dados em sua forma, correção e confiabilidade são de responsabilidade exclusiva dos autores. 


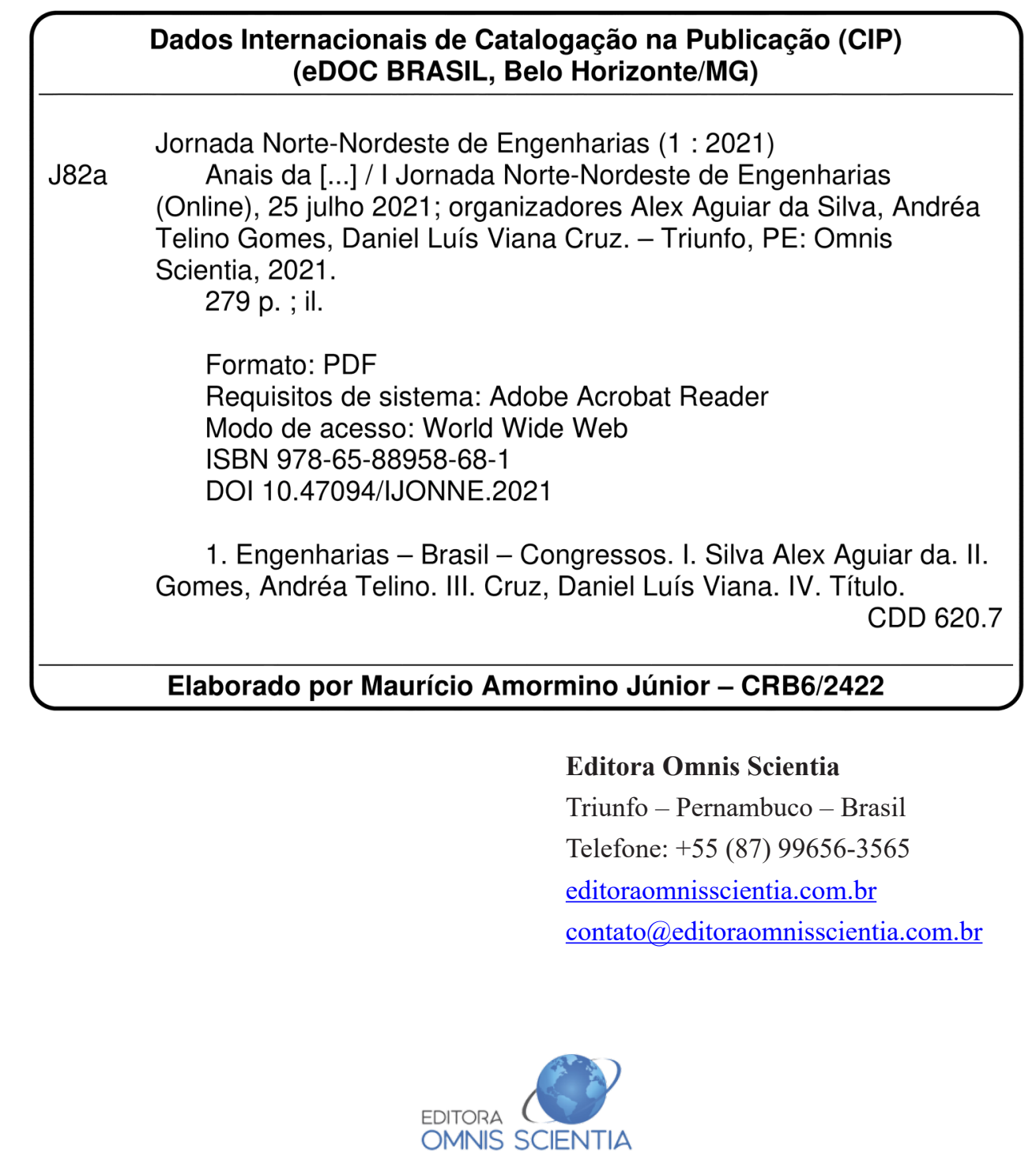




\section{EDITORIAL}

A grande área das engenharias abrangente uma série de ramos específicos, cada qual com determinados campos de aplicação e tipos de tecnologias, conjugando conhecimentos especializados no sentido de viabilizar as utilidades, tendo em conta a sociedade, a técnica, a economia e o meio ambiente. Deste modo, as engenharias aplicam o conhecimento científico, econômico, social e prático, para criar, desenhar, construir, manter e melhorar estruturas, máquinas, aparelhos, sistemas, materiais e processos. Assim, se adquire e se aplicam os conhecimentos matemáticos e técnicos na invenção, aperfeiçoamento e implementação de utilidades que realizem uma função ou objetivo.

A I Jornada Norte-Nordeste de Engenharias (online) I JONNE, objetivou reunir e integrar as engenharias para promover e apoiar esta área do conhecimento por meio de palestras, submissões de trabalhos na modalidade de resumos expandidos e exposição dos resumos aprovados.

Os participantes receberam certificados de participação de 20 horas e foi concedido menção honrosa para os três melhores trabalhos. Seguem os títulos dos resumos que foram premiados:

Modalidade resumo expandido

387210 - DIMENSIONAMENTO DE TROCADOR DE CALOR A PLACAS PARA PASTEURIZAÇÃO DE LEITE PELO PROCESSO HTST

387222 - DESENVOLVIMENTO DE UM DISPENSER DE ÁLCOOL GEL AUTOMÁTICO PARA MONITORAMENTO DA COMPLACÊNCIA DE MÃOS

387225 - REDE NEURAL ARTIFICIAL PARA VISCOSIDADE E CONDUTIVIDADE TÉRMICA DO FLUIDO REFRIGERANTE R32

A equipe organizadora da I JONNE agradece a todos os participantes, palestrantes e avaliadores pela participação e colaboração no congresso. 


\section{SUMÁRIO}

\section{ENGENHARIA CIVIL}

ANÁLISE DO USO DE RESÍDUO DE BRITAGEM DAS ROCHAS EM CONCRETO AUTOADENSÁVEL: UMA REVISÃO DA LITERATURA.....................................................12

ESTRATÉGIAS PARA A PREVENÇÃO DE ACIDENTES NA CONSTRUÇÃO CIVIL: UMA REVISÃO SISTEMÁTICA DA LITERATURA.

RESISTÊNCIA À COMPRESSÃO DE MISTURAS DE SOLO-CIMENTO COM NANOSSÍLICA E SÍLICA ATIVA.

PATOLOGIAS EM OBRAS PARALISADAS: O CASO DO ESTÁDIO COLOSSO DO TAPAJÓS NA CIDADE DE SANTARÉM - PA.

DIFERENÇA NO CUSTO DE MÃO DE OBRA EM FABRICAÇÃO DE LAJES PRÉ- MOLDADAS EM AMBITO NACIONAL

ESTUDO COMPARATIVO DE FUNDAÇÕES PARA UM EDIFÍCIO MODELO EM JOÃO PESSOA/PB: SAPATA X ESTACA HÉLICE CONTÍNUA.

MANIFESTAÇÕES PATOLÓGICAS EM EDIFICAÇÕES COM PAREDES DE CONCRETO ARMADO: ESTUDO DE CASO EM OBRA NA CIDADE DE JOÃO PESSOA - PB.....

REUTILIZAÇÃO DO PÓ RESIDUAL DE MARMORARIA PARA SUBSTITUIÇÃO DO AGREGADO MIÚDO NO CONCRETO.

SUBSIDÊNCIA OCORRIDA NOS BAIRROS PINHEIRO, BEBEDOURO E MUTANGE EM MACEIÓ - AL: UMA REVISÃO BIBLIOGRÁFICA.

CARACTERIZAÇÃO FÍSICA DAS VARIAÇÕES DIMENSIONAIS DO BAMBU GUADUA WEBERBAUERI DE RIO BRANCO - AC.

ESTUDO DA OCORRÊNCIA DE SOLOS COLAPSÍVEIS NA REGIÃO NORDESTE DO BRASIL.

OS EFEITOS DO USO DE POÇOS ARTESIANOS NO PROCESSO DE SALINIZAÇÃO COSTEIRA NA CIDADE DE SÃO LUÍS: UM LEVANTAMENTO BIBLIOGRAFICO.

TEORIADOS SÓLIDOS CELULARES EMPREGADOS EM REFORÇOS PARAACONSTRUÇÃO CIVIL

AVALIAÇÃO DO EXTRATO DE BOLDO E AROEIRA COMO INIBIDOR VEGETAL DE BAIXO CUSTO......

ANÁLISE DE DOSAGEM DE MATRIZ CIMENTÍCIA COM FIBRAS DE POLIPROPILENO: REVISÃO DA LITERATURA 
ANÁLISE DA RETENÇÃO DE CLORETOS EM ARGAMASSAS COM RESÍDUOS DE CELULOSE.

\section{ENGENHARIA DE MATERIAIS E METALÚRGICA}

ANÁLISE DA DEGRADAÇÃO FERRÍTICA DO AÇO INOXIDÁVEL DUPLEX SAF 2205 SUBMETIDO A ENVELHECIMENTO ISOTÉRMICO.

ANÁLISE DE INCLUSÕES E QUANTIFICAÇÃO DE POROSIDADE DO BRONZE AO ESTANHO EM ESTRUTURA BRUTA DE FUSÃO. 100

SÍNTESE RÁPIDA DE NANOFIOS DE NIOBATO DE SÓDIO. 105

AVALIAÇÃODAINCORPORAÇÃODACAREPADELAMINAÇÃOCOMOPARTEDAMATÉRIAPRIMA NA PRODUÇÃO DE SÍNTER DE FINOS DO MINÉRIO DE FERRO. 110

ESTUDO PROSPECTIVO DO PETRÓLEO APLICADO EM NANOMATERIAL CERÂMICO...... 115

ESTUDO PROSPECTIVO DE NANOMATERIAL CERÂMICO APLICADO EM SAÚDE MÉDICA E DENTÁRIA.

ESTUDO PROSPECTIVO DE MAGNETITA NANOMÉTRICA COMO MATERIAL FERROMAGNETICO.

ESTUDO PROSPECTIVO DE CERÂMICA AVANÇADA UTILIZANDO DOPAGEM......

BUSCA DE ANTERIORIDADE DE CERÂMICA APLICADA NA REMOÇÃO DE CONTAMINANTE..... .131

APLICAÇÕES DE $\mathrm{MoO}_{3}$ OBTIVO ATRAVÉS DE PRECIPITAÇÃO. 136

REVISÃO BIBLIOGRÁFICA SOBRE TUNGSTATO DE BÁRIO EM MEIO AQUOSO. 140

ESTUDOS DA DIFUSÃO DE CARBONO NOS AÇOS SAE 1020 E 1045 SUBMETIDOS À TRATAMENTO TERMOQUÍMICO DE CEMENTAÇÃO SÓLIDA. 145

\section{ENGENHARIA DO PRODUTO}

DESENVOLVIMENTO DE UM DISPENSER DE ÁLCOOL GEL AUTOMÁTICO PARA MONITORAMENTO DA COMPLACÊNCIA DE MÃOS. 150

\section{ENGENHARIA ELÉTRICA}

ENERGIA EÓLICA NO CEARÁ .156

\section{ENGENHARIA HIDRÁULICA}

ESTUDO DE MÉTODOS ANALÍTICOS PARA DETERMINAÇÃO DE PERDA DE CARGA EM TUBULAÇÕES DE PVC UTILIZADAS EM IRRIGAÇÕES. 162 
DESENVOLVIMENTO DE PRÁTICAS LABORATORIAIS APLICANDO OS CONCEITOS DE FENÔMENOS DE TRANSPORTES. 168

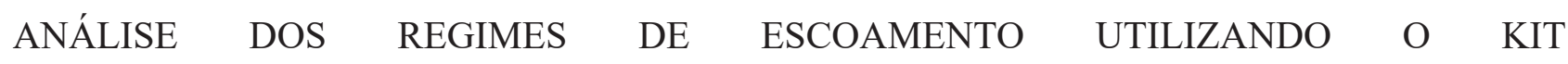
AQUALIBRIUM...

\section{ENGENHARIA MECÂNICA}

AVANÇO NO EMPREGO DE GABARITOS DE SOLDAGEM NO PROCESSO DE FABRICAÇÃO DE CHASSIS FORMULA SAE..

ANÁLISE DA DISTRIBUIÇÃO DE TEMPERATURA NOS PNEUS DE UM VEÍCULO FORMULA SAE PARA DIFERENTES CARGAS VERTICAIS. 184

\section{ENGENHARIA QUÍMICA}

UTILIZAÇÃO DE OXIDAÇÃO AVANÇADA PARA O TRATAMENTO DE EFLUENTES INDUSTRIAIS: UMA REVISÃO DA LITERATURA..

ANÁLISE DOS PRINCIPAIS PROCESSOS DE PRODUÇÃO DE BIODIESEL: UMA REVISÃO DE LITERATURA.

A QUÍMICA DOS AGROTÓXICOS USADOS EM AGRICULTURA: UMA REVISÃO DA LITERATURA. 201

AVALIAÇÃO CINÉTICA DA REAÇÃO DE DEGRADAÇÃO DE DIURON PRESENTE EM ÁGUAS SUPERFICIAIS.

ESTUDO DE CASO DO TRATAMENTO DE GÁS ÁCIDO COM COLUNA DE ABSORÇÃO DE LEITO RECHEADO.

ELABORAÇÃO DA CURVA DE CALIBRAÇÃO PARA ACRILAMIDA PELO MÉTODO DE CROMATOGRAFIA LÍQUIDA DE ALTA EFICIÊNCIA.

DETERMINAÇÃODECONSTANTESCINÉTICASPELOS MÉTODOSINTEGRALEDERUNGEKUTTA PARA REAÇÃO DE OXIDAÇÃO DO ANTRACENO.

DIMENSIONAMENTO DE TROCADOR DE CALOR A PLACAS PARA PASTEURIZAÇÃO DE LEITE PELO PROCESSO HTST.. 229

REDE NEURAL ARTIFICIAL PARA VISCOSIDADE E CONDUTIVIDADE TÉRMICA DO FLUIDO REFRIGERANTE R32.

TRANSFORMAÇÃO DO POLITEREFTALATO DE ETILENO RECICLADO EM FIBRAS TÊXTEIS DE POLIÉSTER. .239

\section{ENGENHARIA SANITÁRIA}

O USO DA ENERGIA SOLAR EM HOSPITAIS EM TEMPOS DE PANDEMIA 
ESTUDO DE VARIÁVEIS QUE POSSAM INFLUENCIAR NO CONSUMO DE ÁGUA DA CIDADE DE BAIXO GUANDÚ-ES........

\section{OUTRAS}

SISTEMAAUTOMÁTICO DE IRRIGAÇÃO. .260

A FRUTA DO MILAGRE - SYNSEPALUM DULCIFICUM.. 266 FOSSAS DE EVAPOTRANSPIRAÇÃO: UMA SOLUÇÃO SUSTENTÁVEL PARA OS DEFICITS NO SANEAMENTO EM ÁREAS CARENTES. .271

PROJETO DE ROBÓTICA SOCIAL E EDUCACIONAL .276 


\title{
DESENVOLVIMENTO DE UM DISPENSER DE ÁLCOOL GEL AUTOMÁTICO PARA MONITORAMENTO DA COMPLACÊNCIA DE MÃOS
}

\author{
André Felipe Krasnievicz da Costa ${ }^{1}$; Gilson Fernandes Braga Junior²; \\ Discente, Universidade Federal do Oeste do Pará (UFOPA), Santarém, PA. \\ Docente, Universidade Federal do Oeste do Pará (UFOPA), Santarém, PA.
}

DOI: 10.47094/IJONNE.2021.6

\begin{abstract}
RESUMO
O simples ato de higienizar as mãos evita que elas sejam veículos de transmissão de doenças, um fato ainda mais atenuante se levado em consideração o momento atual de pandemia do Sars-Cov2 (Covid-19), mas também sendo necessário em locais onde os organismos patogênicos são comuns, como hospitais. Com isso esse projeto tem como objetivo desenvolver um dispenser de álcool em gel automático, no qual evite a transmissão desses organismos, como também ajude no combate ao Covid-19, através da automatização do processo de monitoramento da complacência de mãos, por meio do uso de dispositivos eletrônicos microcontrolados por ESP32, conectado por um circuito ao sensor fototransistor, ao modulo RF-ID que identifica o utilizador por um cartão e a uma bomba. Com o auxílio da plataforma IOT ThingSpeak que faz o monitoramento da utilização dos usuários do dispenser, com recolhimento dos dados de uso com data, hora e quantidade de vezes acionado.
\end{abstract}

PALAVRAS-CHAVE: Dispenser automático. Microcontrolador. Dispositivos eletrônicos.

ÁREA TEMÁTICA: Engenharia do Produto.

\section{INTRODUÇÃO}

As mãos, caso não seja efetuada a higienização correta, são veículos de organismos patogênicos. No contexto da atual pandemia por conta do Sars-Cov2 (Covid-19), uma das medidas mais importantes de prevenção, além da utilização de máscara e realização de distanciamento social, é a correta higienização das mãos. A adesão aos eventos de higienização das mãos é um parâmetro importante a ser avaliado (complacência da higienização de mãos) tradicionalmente em ambiente hospitalar, em que os profissionais da saúde são estimulados, a partir de ações de conscientização e treinamento, a realizarem este procedimento básico, que muitas vezes deixa de ser realizado por diferentes motivos.

Estes motivos consistem por exemplo na falta de adequação do local de instalação do mecanismo 
de higienização (pias, dispensers de álcool gel), falta de tempo do profissional de saúde por conta de carga horária de trabalho excessiva, ou mesmo falta de material à disposição para a realização dos eventos de higienização, que são recomendados não apenas para médicos e enfermeiros, mas também outros funcionários e acompanhantes (RANNIN, Karen P. P., 2016). Este parâmetro de monitoramento pode ser expandido para outros setores da sociedade, como instituições de ensino e empresas no geral, visto que estas medidas devem ser tomadas possivelmente em quaisquer ambientes em que exista trabalho presencial ou semi-presencial.

Com o intuito de facilitar a coleta de dados dos eventos de higienização, dispositivos inteligentes têm sido desenvolvidos, como por exemplo microprocessados, capazes de coletar informações sobre as ações de higienização em lugares específicos (ARRA, A. R., EDMOND, M. B., 2012). Desta forma, propõe-se através deste plano de trabalho o desenvolvimento de um dispenser de álcool gel automático para coleta de informações como quantidade de eventos de higienização e os horários de realização destes eventos, podendo auxiliar o direcionamento de ações de conscientização para este importante procedimento de prevenção da transmissão de doenças.

\section{METODOLOGIA}

O trabalho iniciou-se pela realização de testes para escolha do mecanismo de fornecimento de álcool-gel (mini bombas ou eletroválvula), em protótipos de dispensers desenvolvidos com chapas de PVC. Para isto, foram feitos testes ligando diretamente estes dispositivos em suas fontes apropriadas e foi utilizado um circuito de testes com led infravermelho e foto transistor para acionamento do dispositivo. Em seguida, realizou-se o ajuste do sensor de presença para detectar as mãos do usuário em comunicação com uma plataforma microcontrolada ESP32, e então, foi feita a programação do ESP32 para identificação do usuário por RF-ID. Por fim, foi implementada a comunicação do ESP32 com a plataforma online ThingSpeak, para fornecer informações de utilização do sistema através da internet.

A primeira bomba testada foi uma mini bomba submersa para água, modelo JT100, tensão de 5 volts, com vazão de aproximadamente 1,5 litros por minuto (Figura 1 a)), inserida no interior de um reservatório plástico, com uma mangueira de cristal para saída do álcool gel (Figura 1 b)), com o protótipo para esta bomba na Figura $1 \mathrm{c}$ ). A segunda bomba avaliada foi a bomba elétrica peristáltica - que não tem contato direto com o fluido a ser bombeado -, modelo RS385, com tensão de 12 volts, com vazão de aproximadamente 2 litros por minuto (Figura $1 \mathrm{~g}$ )), posicionada dentro do protótipo (Figura 1 h) e i)). Foi utilizada também uma válvula solenoide simples para água, a mesma usada em lavadoras, com tensão de 12 volts, porém a grade no interior da rosca de entrada de água foi retirada para facilitar a passagem do álcool em gel, que tem uma viscosidade maior que a água (Figura 1 d), vista no protótipo das Figuras 1 e) e f)). Pode ser visto na Figura 1 j) o circuito 3D desenvolvido para teste, consistindo em amplificador operacional para comparação de tensão, para detecção da mão do usuário acionando o dispenser. 
Figura 01 a) Bomba 1; b) Protótipo; c) Protótipo com bomba; d) Eletroválvula; e) Protótipo com eletroválvula; f) Protótipo com eletroválvula com tampa; g) Bomba 2; h) Protótipo com bomba; i) Protótipo com bomba com tampa; j) Circuito

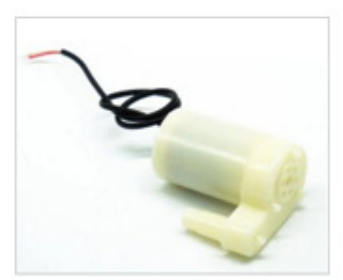

a)

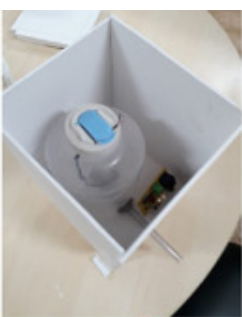

b)

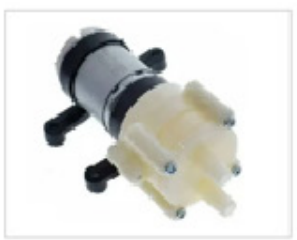

g)

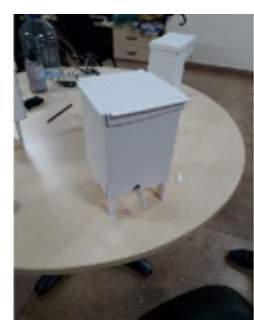

c)

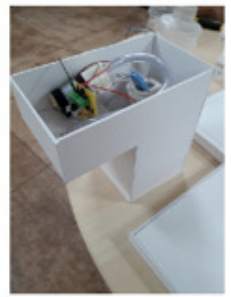

h)

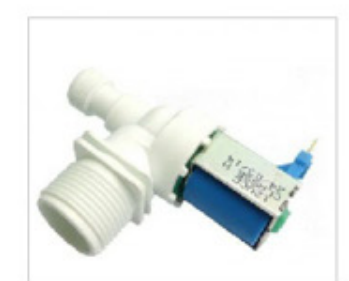

d)

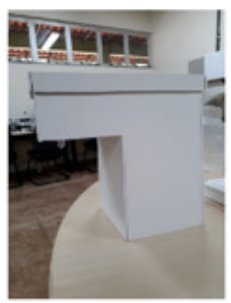

i)

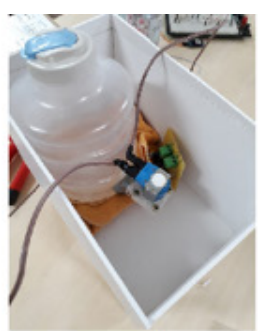

e)

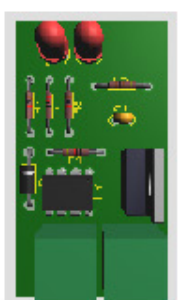

j)

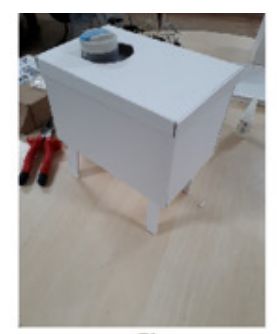

f)

Todas as bombas foram submetidas a testes semanais, sendo acionadas em certo dia da semana, e após uma semana foram acionadas novamente no mesmo dia da semana, por um período de 4 semanas. A válvula elétrica foi ligada diretamente ao reservatório com massa adesiva epóxi e ligada a fonte de tensão. Para ela foi feito um único teste.

Para o circuito com ESP32, escolheu-se o sensor óptico reflexivo fototransistor, modelo TCRT5000, que tem um sensor infravermelho, que funciona como emissor, e um fototransistor, que atua como um receptor, acoplados, facilitando a instalação no dispenser. Para fazer o ajuste de sensibilidade do sensor, foi usado um microcontrolador ESP32, programado com a plataforma Arduino. $\mathrm{O}$ algoritmo carregado ao microcontrolador tem como função ler um valor de tensão analógica e mostrar no monitor serial um valor digital, fornecido pelo sensor de acordo com a distancia em que o obstáculo está posicionado em relação ao mesmo. Uma série de medições foi feita, variando a altura em 1 centímetro, com altura inicial de 1,5 centímetros até altura final de 10,5 centímetros, sendo que para cada altura uma média de 5 valores digitais de tensão foi calculada, para minimizar erros. Após a coleta desses valores foi plotado um gráfico da altura em função do valor do conversor analógico digital (valor inteiro entre 0 e 4095). O ajuste do sensor reflexivo fototransistor resultou nesse gráfico da Figura 02, sendo possível visualizar a altura na qual o sensor vai ser acionado com um valor digital correspondente ao valor analógico de tensão fornecido pelo sensor óptico, e assim fazendo sua calibração. 
Figura 02: a) Gráfico de ajuste do sensor; b) Circuito com microcontrolador e RFID

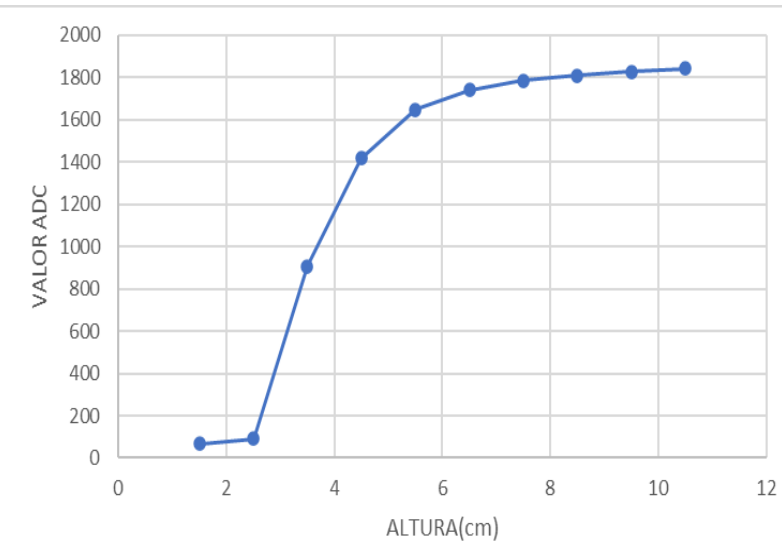

a)

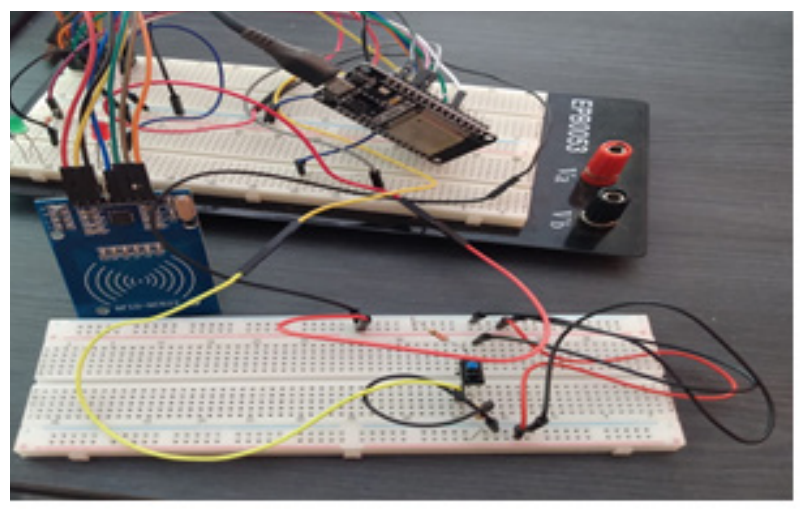

b)

Para fazer o monitoramento do uso do dispenser, foi utilizado a plataforma de desenvolvimento IOT (Internet Of Things) THINGSPEAK ${ }^{\mathrm{TM}}$, de forma que os dados coletados pelo equipamento são enviados para nuvem e tratados em forma de gráfico, com data e horário em que houve o acionamento do mesmo.

\section{RESULTADOS E DISCUSSÕES}

A partir dos experimentos feitos, constatou-se que a bomba 1, mini bomba submersa, não serve para o bombeamento de álcool em gel, já que apresentou uma vazão bem abaixo do esperado e parou de funcionar após uma semana, devido a entupimento, por ressecamento do líquido. Já a bomba 2, bomba peristáltica, obteve resultados positivos e apresentou capacidade de bombear um fluido tão viscoso como o álcool em gel, sem dificuldades, mas com uma vazão maior que a necessária para o devido fim, sendo preciso alterar a tensão de funcionamento para uma menor que 12 volts usados inicialmente, para uma de aproximadamente 9 volts. A válvula solenoide foi necessário apenas um teste para constatar que não serviria ao propósito, pois não teve vazão alguma do líquido, não precisando dar continuidade aos testes e descartando a possibilidade de seu uso.

Com o RF-ID foi possível fazer a identificação do usuário, por meio de 2 cartões, contendo em cada um seu código de identificação e gravado o nome do proprietário, mas também identificando quando o utilizador não tiver cartão. Sendo estabelecida a comunicação com o ThingSpeak da forma descrita anteriormente, os dados de utilização do dispenser puderam ser separados e gravados por usuário identificado, gerando seus próprios resultados de uso, quantidade de vezes que acionou, horário que usou e dia, como também por usuário não identificado. Até o presente momento de submissão deste resumo, o gráfico gerado pela plataforma se deu, ainda, somente para usuário não identificado, resultando no gráfico da figura 03. 
Figura 03: Gráfico de utilização

Quantidade acionado usuário sem cadastro

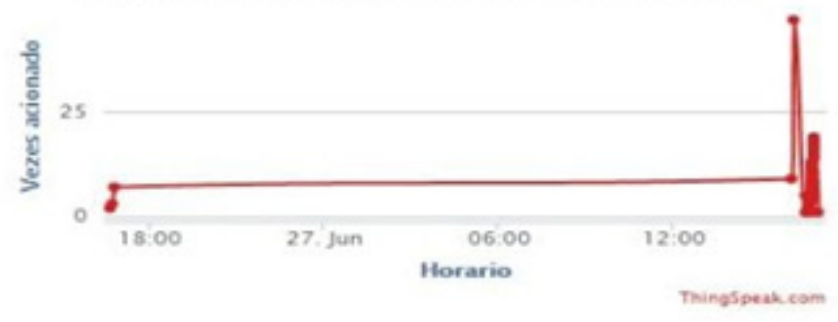

\section{CONSIDERAÇÕES FINAIS OU CONCLUSÃO}

Conclui-se que a proposta do presente trabalho é desenvolver um dispenser de álcool gel inteligente, para auxiliar as pessoas de forma prática na complacência de mãos, através do uso de chapas de PVC para construir a estrutura e dispositivos eletrônicos microcontrolados por um ESP32 e conectados a plataforma IOT ThingSpeak que integram a parte física do dispenser. Apesar dos resultados serem satisfatórios, nota-se a necessidade de melhorar o protótipo, que apresenta mal funcionamento como acionamentos aleatórios e até a falta de comunicação, como também o circuito, que se desconecta facilmente dos furos da protoboard e além disso dar continuidade ao projeto fazendo com que seja possível recolher dados dos usuários identificados por cartão, além da inclusão de um sistema de monitoramento de nível mínimo do dispenser.

\section{PRINCIPAIS REFERÊNCIAS}

RANNIN, Karen P. P., CAMPANHARO, Cássica R. V., LOPES, Maria C. B. T., OKUNO, Meiry F. P., BATISTA, Ruth E. A. Adesão à higiene das mãos: Intervenção e avaliação. Cogitare Enferm, 2016 Abr/Jun; 21(2): 01-07.

ARRA, A. R., EDMOND, M. B. Hand Hygiene: State-of-the-Art Review With Emphasis on New Technologies and Mechanisms of Surveillance. Curr Infect Dis Rep 2012; 14:585-591. 


\section{editoraomnisscientia@gmail.com M} https://editoraomnisscientia.com.br/

@editora_omnis_scientia @ https://www.facebook.com/omnis.scientia.9 f

$$
\text { +55 (87) 9656-3565 @ }
$$




\section{editoraomnisscientia@gmail.com M} https://editoraomnisscientia.com.br/ $\oplus$

@editora_omnis_scientia@ https://www.facebook.com/omnis.scientia.9 f

$$
\text { +55 (87) 9656-3565 @ }
$$

